\title{
LINEAMENTI GEOMORFOLOGICI DELLA COSTA E DEI FONDALI DEL GOLFO DI TRIESTE E CONSIDERAZIONI SULLA LORO EVOLUZIONE TARDO-QUATERNARIA ${ }^{\circ}$
}

\author{
Ruggero Marocco *
}

\section{GENERALITĀ}

Il Golfo di Trieste è uno specchio d'acqua poco profondo (profondità massima di circa $25 \mathrm{~m}$ ) ubicato nella parte nord-orientale del Mare Adriatico. $\grave{E}$ contornato via via in senso antiorario dalle coste alte della penisola istriana, dall'altopiano carsico e quindi dalle coste basse, definite da una successione di delta e lagune connesse alla piana alluvionale dei principali fiumi veneto-friulani e, in particolare ad oriente dall'Isonzo e ad occidente dal Tagliamento. Verso Ovest è aperto al Golfo di Venezia.

Territorialmente è diviso in una parte italiana, a settentrione della mid-line definita dagli accordi bilaterali del 1968, e in una parte yugoslava che contorna l'Istria.

È soggetto all'influsso di notevoli apporti di acque continentali provenienti soprattutto dal versante italiano (fiumi Tagliamento $90 \mathrm{mq} / \mathrm{sec}$, Isonzo $170 \mathrm{mq} / \mathrm{sec}$, quindi Timavo, Rosandra e Ospo) e, subordinatamente, dai fiumi Risano e Dragogna nel versante istriano. Gli apporti solidi sono per la maggior parte provenienti dai fiumi Tagliamento e Isonzo e assommano complessivamente a $4 \times 10^{6} \mathrm{mq}$ all'anno (dati Regione FriuliVenezia Giulia), con una preponderanza di sedimenti fini su quelli sabbiosi.

La circolazione delle acque è determinata prevalentemente da correnti di gradiente con andamento generale ascendente lungo le coste istriane e verso Ovest lungo quelle veneto-friulane, a cui si sovrappongono le correnti di marea ma soprattutto quelle di deriva determinate dalla Bora (ENE), vento regnante e dominante nel paraggio.

* Istituto di Geologia e Paleontologia dell'Università di Trieste.

- Lavoro eseguito con il contributo finanziario del programma di ricerca M.P.I. $60 \%$ "Geomorfologia ed evoluzione del Friuli-Venezia Giulia”, responsabile F. Vaia. 
Il Golfo di Trieste è caratterizzato da una notevole oscillazione del livello marino determinata da maree di tipo semidiurno (tra le maggiori del Mediterraneo) con un'escursione media a Trieste di $86 \mathrm{~cm}$ in sizigie e di $\mathrm{cm} 22$ in quadratura. In casi eccezionali (acque alte) si verificano maree che possono anche raggiungere altezze di $\mathrm{cm} 200$ sopra il l.m.m.

Soprattutto lungo la costa alta dell'Istria e dell'altopiano carsico è presente una serie di promontori e baie (Pirano, Capodistria, Muggia e Panzano), sedi di importanti strutture portuali ed industriali. Le coste basse del versante italiano sono, salvo rare eccezioni, difese da una serie quasi continua di opere parallele e trasversali, venutesi ad accumulare in un lasso di tempo di circa 80 anni per iniziative non coordinate. Gli insediamenti urbani, che presentano quasi sempre un nucleo storico, sono stati oggetto di intensi sviluppi, soprattutto durante il periodo postbellico, in funzione turistico-balneare. Oggi tali insediamenti si presentano con struttura continua a nastro immediatamente a ridosso della linea di riva, determinando un ulteriore degrado dei litorali.

\section{IL SOTTOSUOLO}

Scarse sono le informazioni sulla coltre sedimentaria della bassa pianura friulana e del Golfo di Trieste. I dati più interessanti sono forniti da MARTINIS (1953 e 1957) e riferiscono sulla stratigrafia dei pozzi d'acqua profondi rispettivamente di Grado e Lignano, e più recentemente da MOSETTI (1980), ALBRECHT \& MOSETTI (1987) per i terreni a ridosso del Carso monfalconese.

Lo spessore della coltre sedimentaria varia dai $180 \mathrm{~m}$, immediatamente ad Ovest dell'altopiano carsico, a $258 \mathrm{~m}$ a Grado (MARTINIS, 1953) fino a raggiungere circa i $500 \mathrm{~m}$ sul delta del fiume Tagliamento (Pozzo Cesarolo - AGIP). Il substrato è costituito da depositi flyschoidi eocenici che poggiano sui calcari mesozoici; è terrazzato in prossimità di Miramare con piani ubicati a diverse profondità (ALBRECHT \& MOSETTI, 1987). Procedendo verso Ovest è disarticolato in una serie di alti e bassi strutturali legati ad una serie di faglie ad andamento essenzialmente N-S che dal Carso Triestino (FINETTI, 1967) si succedono fino al Tagliamento (Pozzo Cesarolo - AGIP).

Sulla base delle associazioni microfaunistiche riportate da MARTINIS per i sondaggi di Lignano (op. cit.) si è tentata una ricostruzione paleoambientale dell'area, prendendo spunto soprattutto dai lavori di FAVERO \& PASSEGA (1975) condotti sul pozzo C.N.R. Venezia 1, ubicato nell'omonima laguna. La sequenza sedimentaria (Fig. 1) riflette una serie di proces- 

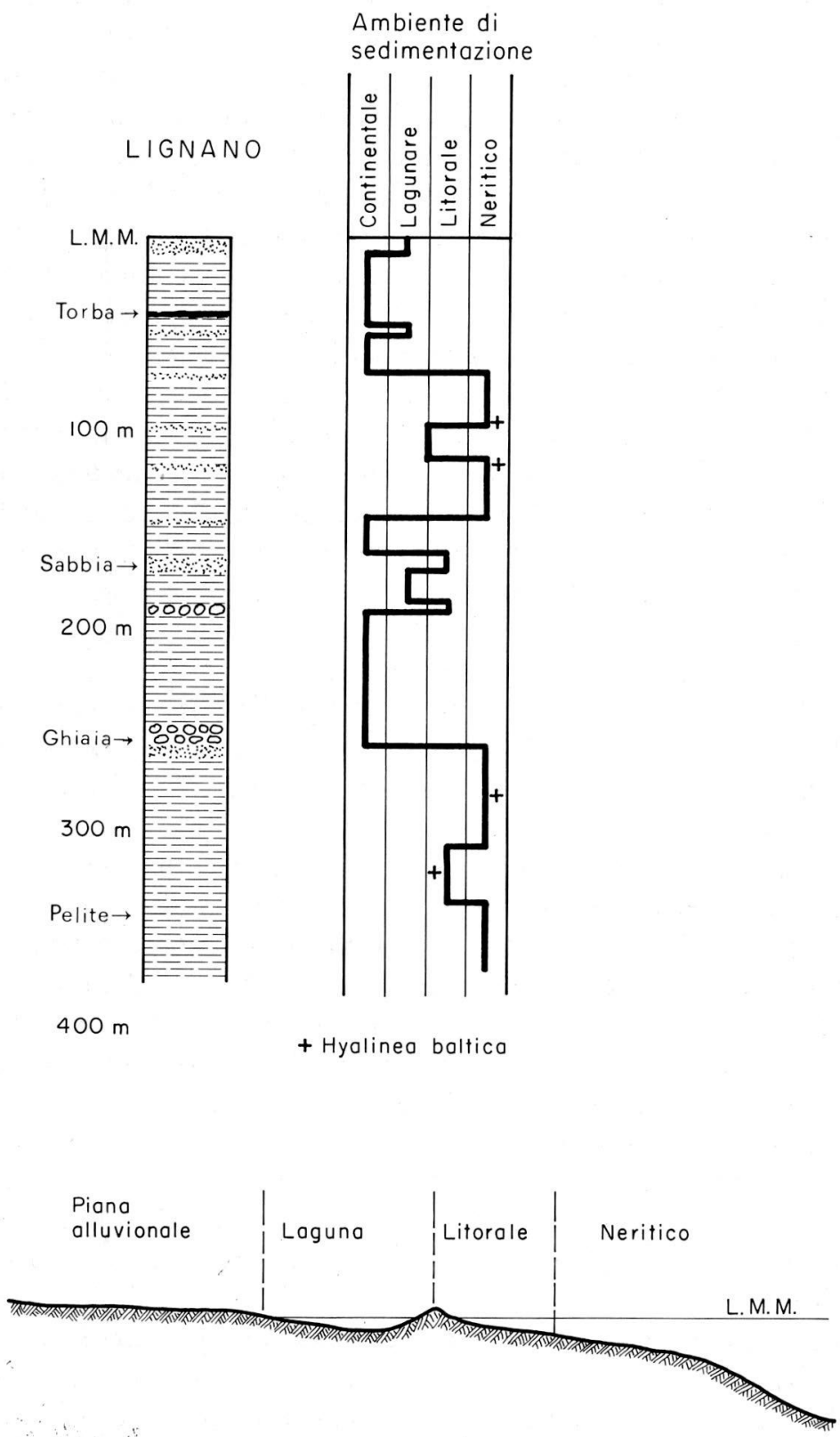

Fig. 1 - Ricostruzione paleoambientale del sottosuolo di Lignano. 
si trasgressivi e regressivi che hanno portato il mare almeno per due volte a sorpassare l'attuale linea di costa del Friuli-Venezia Giulia.

Mancando qualsiasi riferimento cronologico, l'attribuzione della sequenza sedimentaria marina ad eventi trasgressivi legati a fasi interglaciali non è certamente automatica, come d'altra parte non è automatico il collegamento delle fasi di alluvionamento, visualizzate dalla presenza di depositi continentali, con periodi glaciali in generale o specifici in particolare. E questo anche in ragione delle indicazioni climatiche dedotte dalla fauna a Foraminiferi planctonici che evidenziano una persistenza di condizioni fredde delle acque nella gran parte dei sedimenti marini. Si è tentato anche di analizzare i sondaggi riportati dalla letteratura per il sottosuolo della bassa pianura friulana (Bonifica Fraida e Piancada, FERUGLIO, 1936; LIPPARINI, 1936 - Grado, MARTINIS, 1957 - Monfalcone, MOSETTI, 1980; ALB́RECHT \& MOSETTI, 1987) e di correlare i depositi marini con i terrazzamenti rinvenuti sul sottofondo dell'area marina di Miramare. I risultati, come d'altra parte era da attendersi, non hanno risposto alle aspettative in quanto si è verificata l'impossibilità di una soddisfacente correlazione fra i sondaggi. E ciò soprattutto a causa della scarsità dei dati, ma anche dalle frequenti variazioni laterali di facies che non permettono l'individuazione di un «datum» di riferimento.

\section{IL FONDO MARINO}

Le conoscenze acquisite sulla morfologia e natura del fondale del Golfo di Trieste permettono oggi di descrivere minuziosamente il territorio delimitato dalle acque nazionali, e solamente in parte quello attualmente ubicato all'interno delle acque yugoslave. Ciò soprattutto grazie ad una serie di ricerche specifiche eseguite dall'Osservatorio Geofisico Sperimentale di Trieste a cavallo degli anni 1960-70 e compendiate in parte da MOSETTI (1966) e, per quanto attiene alla sedimentologia dei fondali, dall' Istituto di Geologia di Trieste, sintetizzata nella Carta Sedimentologica dell'Alto Adriatico (BRAMBATI et al., 1988).

Il prodotto delle ricerche sulla morfologia del fondo del Golfo di Trieste è riassunto in una carta morfologica-batimetrica redatta da ROSSI, MOSETTI, CESCON (1968) e riportata in forma semplificata in. Fig. 2. I fondali scendono rapidamente in prossimità della costa rocciosa dell'Istria e del Carso Triestino, mentre, lungo le coste basse sabbiose del litorale veneto-friulano, degradano dolcemente con isobate sub-parallele alla costa. Ad eccezione dell'area contraddistinta dalla presenza del Banco sabbioso della Mula di Muggia, che si estende dalle immediate vicinanze della 


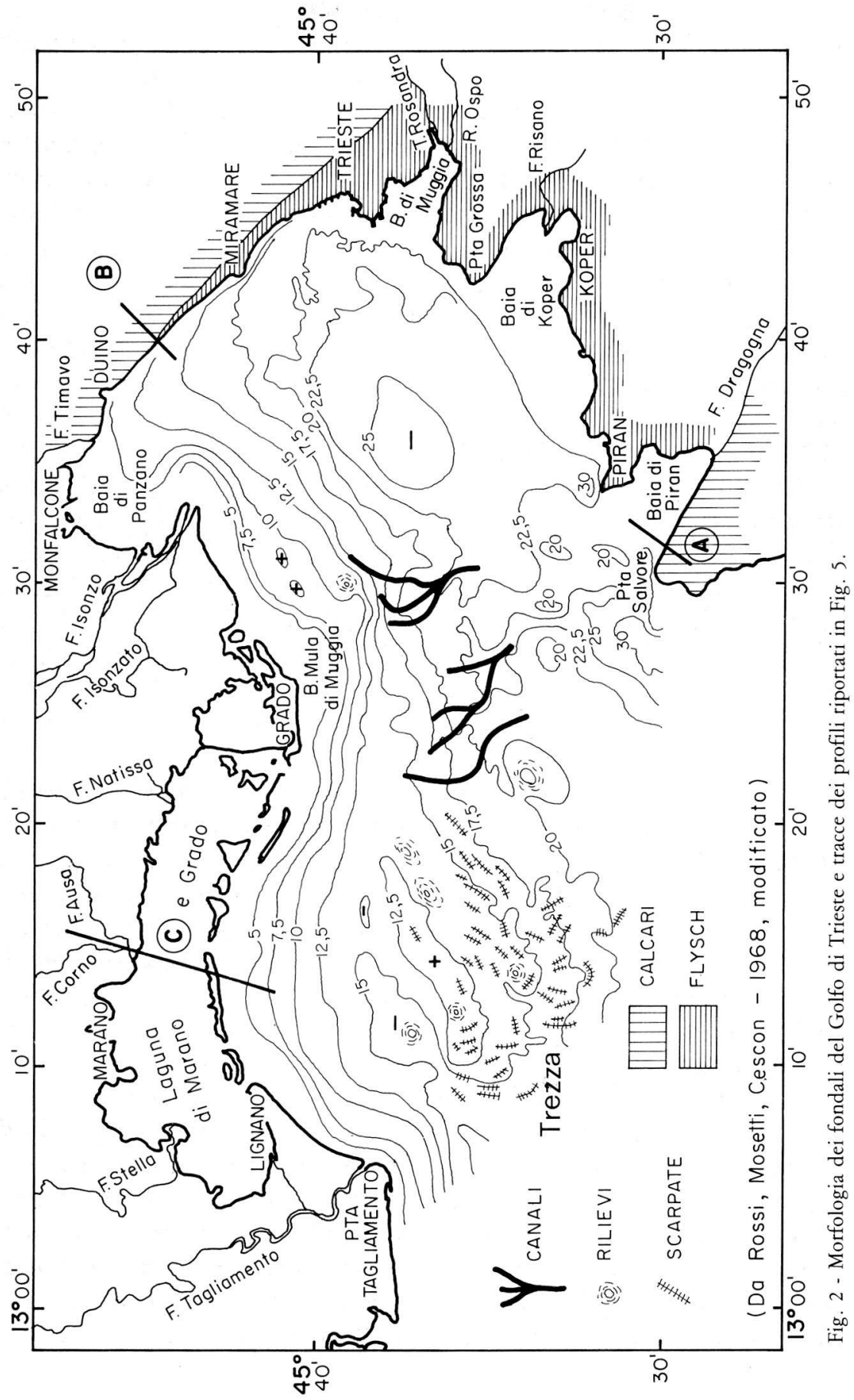


riva del litorale gradese fino alla profondità di circa $15 \mathrm{~m}$, le pendenze sono di circa $3 \mathrm{~m} / \mathrm{km}$ fino alla profondità di $11-12 \mathrm{~m}$. Nell'ambito di questa fascia, e precisamente alla profondità di $2-5 \mathrm{~m}$ si sviluppano sistemi di barre molto irregolari sia per estensione che per numero (2-4 ordini di barre).

Oltre la profondità di 11-12 $\mathrm{m}$ il fondo marino assume caratteri morfologici molto articolati su lievissima pendenza con elevazioni e depressioni dell'ordine di 1-2 $\mathrm{m}$ dal fondo. Le prime sono presenti con maggior frequenza tra Lignano e Grado, le seconde da Grado e Trieste. Le elevazioni del fondo marino sono determinate sia da accumuli di sabbia (sand waves), ma soprattutto da sollevamenti irregolari determinati da "matter a Posidonia" (STEFANON, 1984), particolarmente evidenti alle minori profondità (Fig. $3 \mathrm{~A}$ ) e da formazioni rocciose distinte da NEWTON \& STEFANON (1982) in tre categorie:

- beachrock: si presentano in affioramenti estesi (fino a centinaia di m) e con elevazioni dal fondo di $5-30 \mathrm{~cm}$;

- reef: rocce organogene costituite da alghe calcaree (Lithophyllum, Peyssonelia e Lithothamnium) associate a briozoi e serpule. Hanno forma pinnacolare con elevazioni massima dal fondo di 1-2 m;

- arenarie calcaree: con evidente stratificazione parallela e/o incrociata. Si presentano in blocchi isolati, con elevazioni dal fondo di $1-3 \mathrm{~m}$ (Fig. 3 B).

Altri studi, tra cui BOLDRIN (1979), hanno messo in evidenza come le formazioni definite "beachrock" siano in realtà costituite da due tipi litologici: una roccia clastica sottostante e una roccia organogena soprastante (Fig. 3 C). Non ci è dato di sapere se la roccia sottostante "l'organogeno" sia di origine continentale o litorale (beachrock s.s.). Per dar risposta a questo quesito è stata intrapresa una serie di ricerche, attualmente in corso di svolgimento.

Gli affioramenti rocciosi sono per la quasi totalità ubicati nel tratto di mare a cavallo tra Lignano e Grado, in un alto morfologico nominato localmente "Trezza" (Trave).

Le depressioni presenti soprattutto nella parte orientale del Golfo, o assumono deboli forme circolari (di fronte al delta dell'Isonzo) o forme allungate, sinuose di direzione N-S (particolarmente evidenti di fronte a Grado). Queste ultime, secondo ROSSI et al., (op. cit.) presentano forme e dimensioni tali da far supporre "un residuo di un'antica idrografia emersa" che, sia la trasgressione olocenica, sia la sedimentazione marina successiva non ha completamente cancellato. 

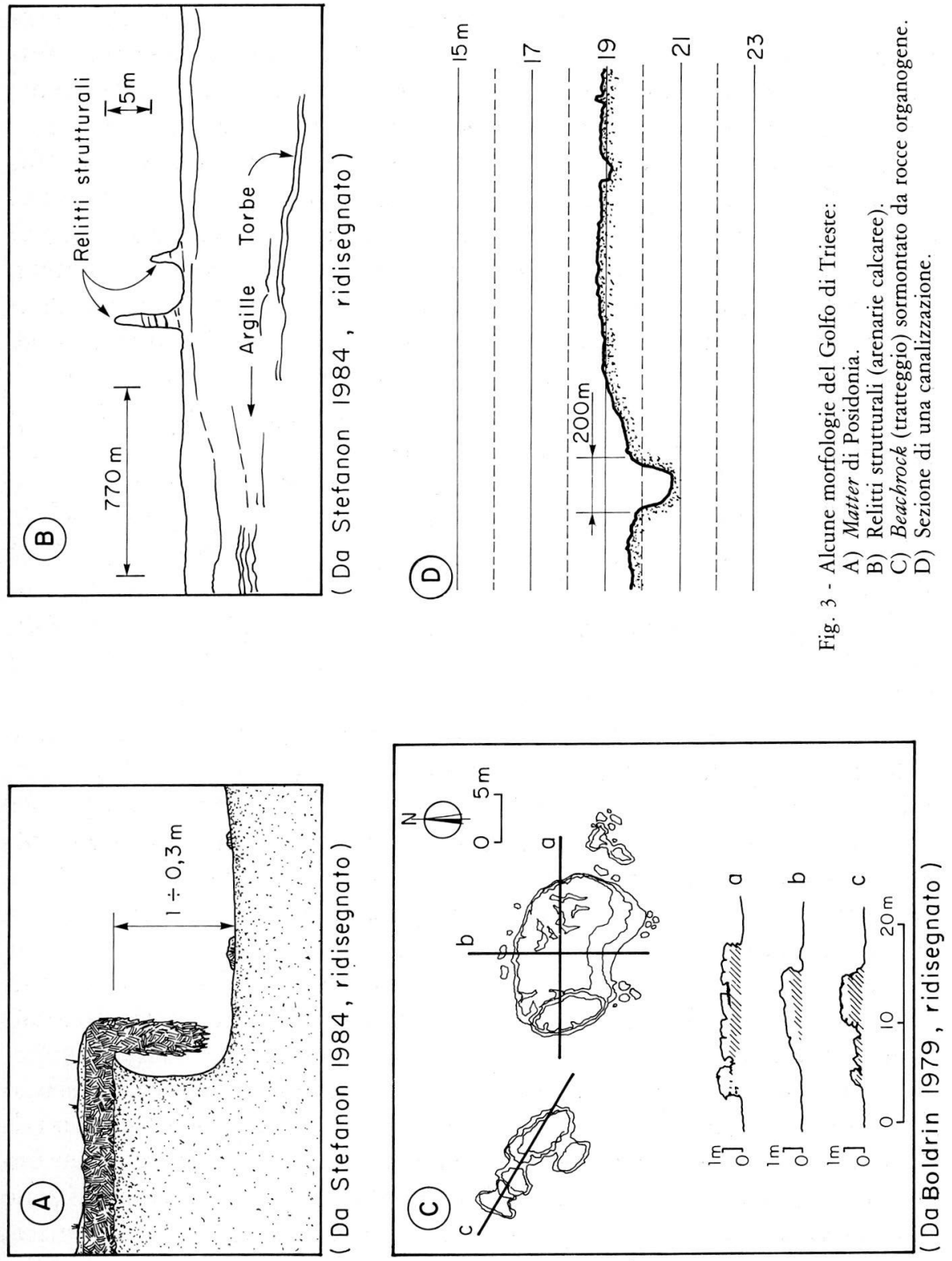
La ripetizione dei rilievi batimetrici nell'area eseguita recentemente, ha evidenziato che le depressioni hanno dimensioni e geometrie che, almeno ad una prima analisi, non trovano riscontro con quelle degli attuali canali lagunari o percorsi fluviali della bassa pianura (ad eccezione delle cosiddette bassure di risorgiva), mentre sono ricorrenti come strutture sepolte nel sottofondo marino (STEFANON, 1984). Anche in questo caso è stata promossa una ricerca che, partendo da dettagliati rilievi batimetrici, ecografici a bassa frequenza, campionature di sedimenti e rilevamento in immersione, dovrebbe dar risposta al problema. A titolo di esempio, nella Fig. 3 D viene riportata una sezione di una depressione a percorso sinuoso. La larghezza del canale è di circa $200 \mathrm{~m}$, con una profondità di $1 \mathrm{~m}$ circa. Oltre queste incisioni che si possono considerare principali, sono presenti, apparentemente in forma ramificata, canalizzazioni secondarie con larghezza di sezione di gran lunga superiore alla profondità (rapporto 300-500 a 1).

La distribuzione dei sedimenti (Fig. 4) riflette da un lato i processi sedimentari che si attuano nell'area, dall'altro gli effetti erosivi della trasgressione marina post-wurmiana. I primi si esplicano essenzialmente lungo la fascia costiera e investono gli apporti terrigeni dei principali fiumi dell'area (e in particolare quelli attuali e subattuali del F. Isonzo) oltre che gli antichi depositi costieri ripresi dal moto ondoso e dalle correnti marine. I secondi sono riconoscibili al largo della fascia costiera, dove secondo più autori, emergono "sedimenti residuali". o meglio sedimenti esumati dalla trasgressione postglaciale. Dal punto di vista tessiturale i sedimenti più fini, pelitici, contraddistinguono gran parte del tratto orientale del Golfo (Baia di Panzano e Trieste) mentre ad Ovest sono relegati alla sola foce del F. Tagliamento. Complementarmente, quelli sabbiosi (o ghiaiosi in corrispondenza delle coste alte) caratterizzano la ristretta area costiera del primo tratto del Golfo, mentre ad Ovest si estendono su ampi tratti costieri (vedi Banco della Mula di Muggia), ma soprattutto nella parte centrale (sabbie di piattaforma).

Nel dettaglio la posizione dei sedimenti evidenzia la classica distribuzione tessiturale da riva verso il largo con sabbie (o ghiaie) costiere seguite, dopo una breve zona di transizione, da sedimenti pelitici che rappresentano l'accumulo dei materiali fini portati in mare dai principali fiumi e aventi una distribuzione areale legata alla dispersione risultante dalle condizioni fluviali e meteomarine. Gli alti morfologici di centro Golfo sono per la quasi totalità costituiti da sabbie, con notevole contenuto detritico, coperte da un velo di pelite che rappresenta la tipica sedimentazione al largo della fascia costiera. 


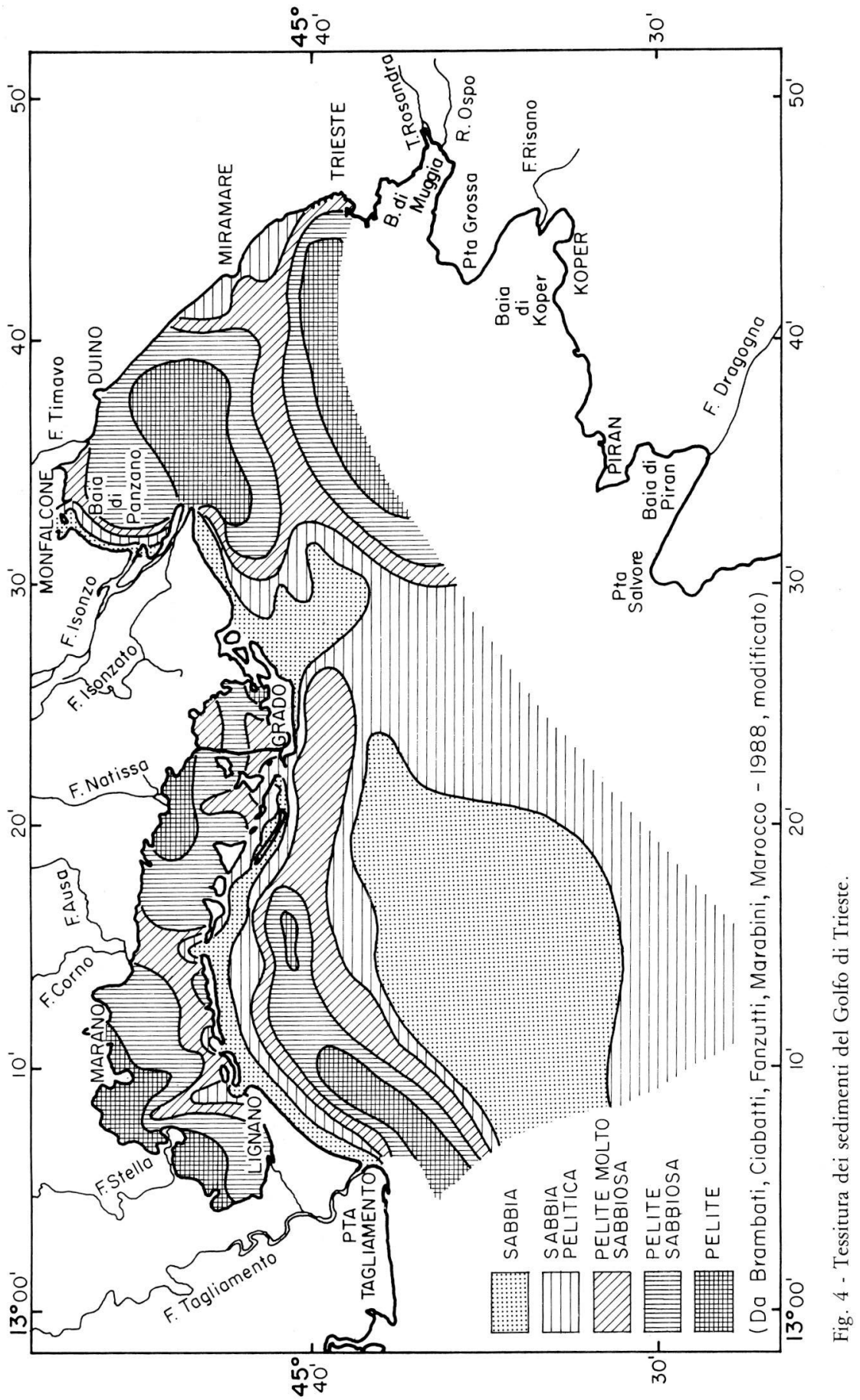


Lungo la fascia litorale della costa bassa del Golfo di Trieste i sedimenti olocenici, marini e lagunari, raggiungono le maggori potenze riscontrate nell'intera area (8-10 m circa) e diminuiscono progressivamente verso il largo formando un prisma sedimentario. Al di sotto del prisma, quasi sempre con contatto erosivo, sono presenti depositi alluvionali wurmiani o postwurmiani che si raccordano con i fondali del centro Golfo. Qui la sedimentazione marina è, come già detto, minima o a volte assente o erosa, cosicché emergono i depositi continentali sottostanti.

A questo proposito va ricordato che, al contrario di quanto si immaginava un tempo, i fondali del Golfo non sono da considerarsi stabili. E ciò sia perché possono essere erosi, trasportati e risedimentati da eventi eccezionali (CAVALERI \& STEFANON, 1980), sia perché sono oggetto di un'intensa elaborazione da parte dei mezzi meccanici dei pescatori di vongole, canolicchi e tartufi. Ricerche attualmente in corso dovrebbero dar risposta al primo dei due problemi, attraverso una definizione qualiquantitativa del materiale sabbioso che si muove al fondo, grazie anche all'utilizzo di trappole batometriche di recente costruzione (TUNIS et al., 1982).

A conclusione di questo capitolo va sottolineato che nelle aree a sedimentazione pelitica attuale la rata di sedimentazione varia notevolmente da luogo a luogo. Recenti studi eseguiti dall'Enea Disp. a seguito dell'incidente di Chernobyl e finalizzati al monitoraggio dei radionuclidi artificiali (soprattutto Cs) nel Golfo di Trieste, hanno evidenziato che l'area più orientale del Golfo, che beneficia degli apporti fini del F. Isonzo, presenta un rateo di sedimentazione di gran lunga maggiore del tratto occidentale, ove prevale una non sedimentazione o addirittura un'erosione dei depositi tilaventini (BALDI et al., in stampa).

Nell'ambito dell'area di influenza degli apporti fini del F. Isonzo non deve essere dimenticato, anche se difficilmente quantificabile, l'apporto dei sedimenti organici (faecal pellets) determinato dagli impianti di mitilicoltura che bordano il Carso Triestino. Rilievi sotto i filari di mitili hanno reso manifesto, anche se in forma discontinua, caotici accumuli di faecal pellets alti fino a $20 \mathrm{~cm}$ dal fondo sconnesso circostante (MAROCCO et al., in stampa).

\section{LE COSTE}

Il settore orientale e meridionale del Golfo è definito da coste alte, a falesia, costiuite da calcari (nel solo tratto Marina di Aurisina - Duino; Fig. 2) ma soprattutto da flysch. Le spiagge sono ridottissime, impostate su ter- 
reni di abrasione e alimentate dall'erosione delle rocce affioranti oppure su depositi deltizi dei corsi d'acqua che incidono il litorale. Profili tipici di questo tratto di costa sono riportati in Fig. 5 (A e B).

Le falesie non evidenziano morfologie legate alla permanenza del livello marino ad una determinata quota (solchi di battente) e ciò soprattutto a causa dell'ampia escursione di marea (Fig. $5 \mathrm{~A}$ ). È invece riconoscibile e ben evidente una zonazione biologica che varia in funzione del l.m.m., ma anche dell'esposizione della costa ai mari regnanti e dominanti (Fig. 6) (TORUNSKI, 1979). Una serie di ricerche (vedi SPENCER, 1988; TORUNSKI, 1979) ha messo in evidenza l'attività di bio-erosione della costa svolta dalla Patella coerules e Littorina neritoides. Complessivamente l'erosione marina globale (abrasione + erosione biologica) che si verifica avviene soprattutto nella fascia intertidale è stata stimata essere pari a circa $0.63 \mathrm{~mm} /$ anno (TORUNSKI, 1979).

Il settore orientale del Golfo (Figg. $5 \mathrm{C}$ e 7) è costituito da spiagge sabbioso-pelitiche e comprende una successione di delta e lagune. Mineralogicamente tutto il litorale fino all'attuale delta del F. Tagliamento dipende dagli apporti terrigeni dei fiumi del sistema isontino (Isonzo, Natisone e Torre). E ciò a dimostrazione che gran parte della pianura venetofriulana ha avuto origine dalle loro divagazioni. A questo proposito va ricordato che su quelle dell'Isonzo a partire dal XVIII secolo esiste una ricca bibliografia che comprende i lavori di DESIO (1922), per concludere con quelli di BRAMBATI (1970 e lavori successivi). In sintesi (Fig. 8) l'Isonzo metteva foce fino al 1730 circa in località Golamento (posizione I), quindi in località Sdobba (posizione II) fino al 1896 e poi, proseguendo sempre la sua diversione verso Est, in località Quarantia (III). Qui il percorso del fiume fu sbarrato in quanto il delta sottomarino andava ad ostruire l'accesso al porto industriale di Monfalcone, e riportato alla posizione attuale (Sdobba, IV) nel 1937.

Attualmente il delta, che può considerarsi stabilizzato, presenta una forma digitata con un unico canale distributore: la piana deltizia subaerea è definita da paludi erbacee e fragmiteti, quella inferiore da piane di marea a sedimentazione sabbioso-pelitica, che si estendono per circa $700 \mathrm{~m}$ attorno alla linea di riva.

Il fronte deltizio è caratterizzato da una serie di barre che a volte confluiscono a formare banchi sabbiosi emergenti alla sinistra della foce. Ecco ora alcuni dati sull'entità dell'arretramento e della progradazione fluviale delle più recenti fasi di diversione. Al momento dello spostamento della foce da Golamento (posizione I di Fig. 8) a Sdobba (posizione II di Fig. 8), 

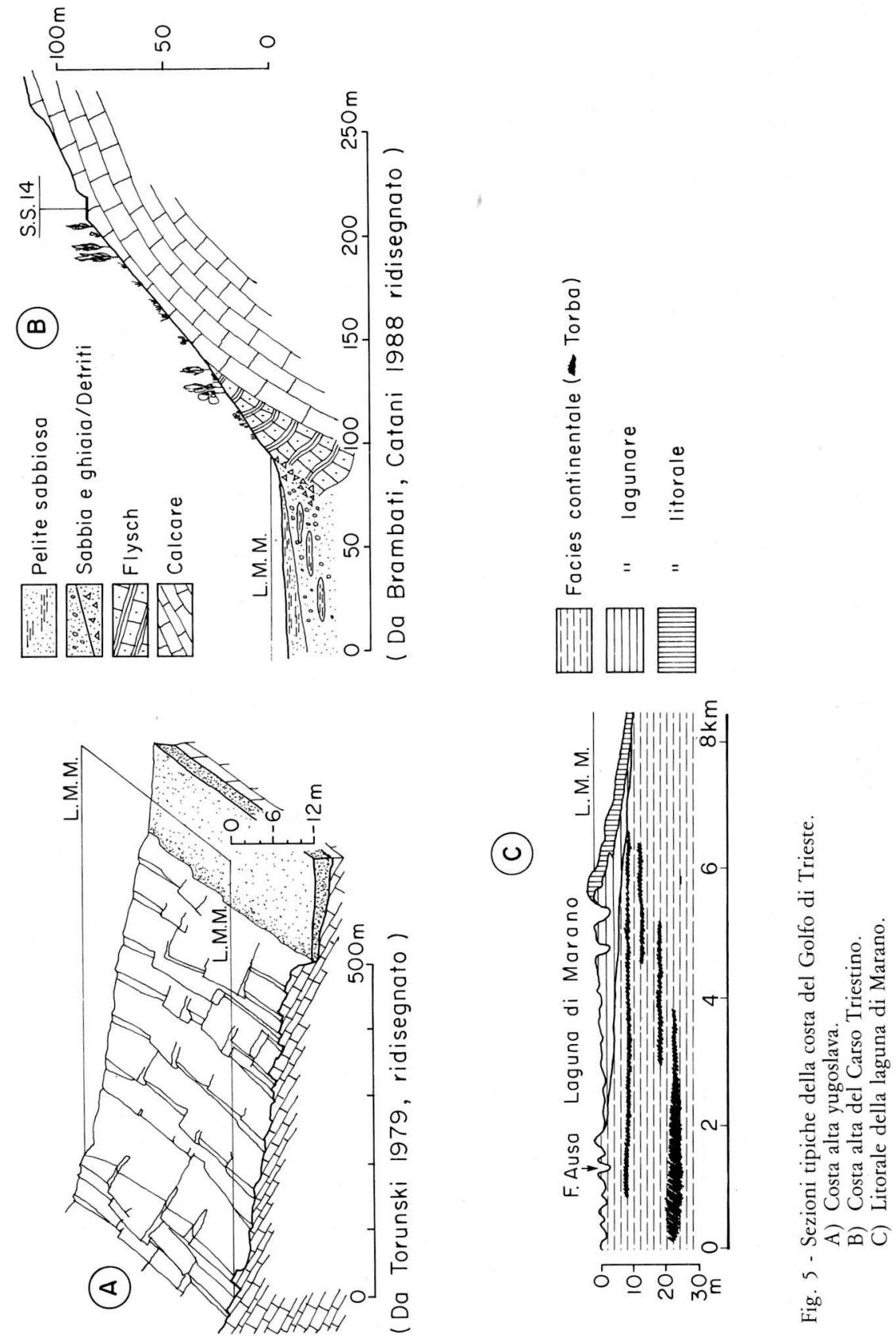


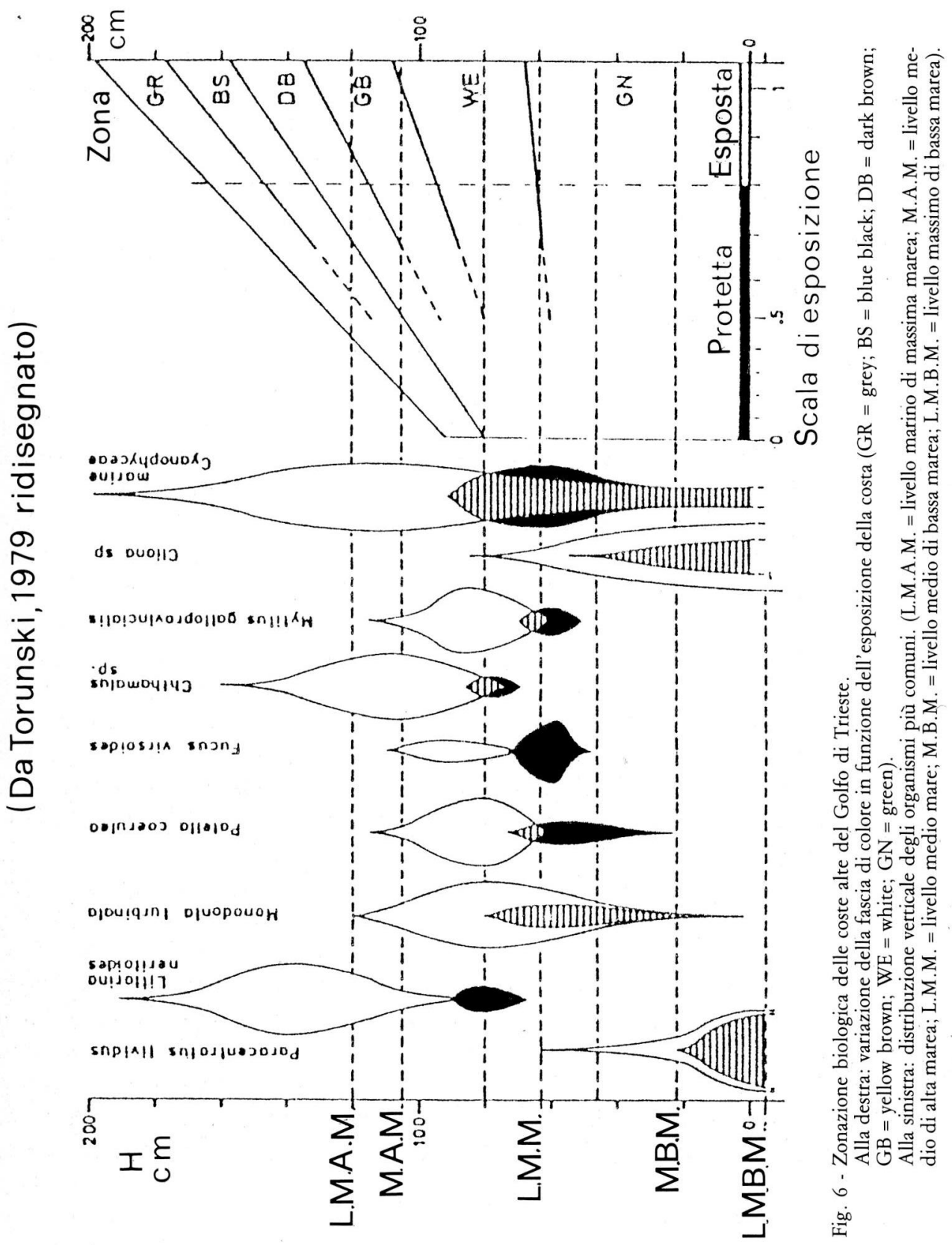


l'area dell'antico delta ha manifestato un arretramento medio di circa 3.4 $\mathrm{m} /$ anno, con punte massime di $5.7 \mathrm{~m} /$ anno e minime, al ritorno dell'Isonzo nello Sdobba, di $2.2 \mathrm{~m} /$ anno. Nel contempo, l'accrescimento medio del delta sommerso può essere valutato su valori di 2.5-3.0 m/anno (dato ricavato da rilievi batimetrici storici editi dalla Regione Friuli-Venezia Giulia). Come detto, oggi tutto il delta e gran parte del litorale adiacente è stabilizzato da opere longitudinali aderenti, integrate da opere trasversali (pennelli). L'immediato retroterra è stato bonificato (Bonifica del Brancolo, di Isola Morosini, della Vittoria e di Fossalon). Il fiume è sede di intense attività estrattive, soprattutto di materiali ghiaiosi e sabbiosi.

Più ad Ovest, al di là della laguna di Marano e Grado, si estende il delta del F. Tagliamento, classica forma bilobata con base di circa $14 \mathrm{~km} \mathrm{e}$ lati corrispondenti ai lidi di Lignano ad Est e Bibione ad Ovest, rispettivamente di 7 e $9 \mathrm{~km}$ circa. Il corpo del delta è costituito da un'impalcatura di rilievi sabbiosi allungati (almeno in numero di otto), separati tra loro da una leggera depressione, spesso a carattere acquitrinoso (lama).

Gran parte di questi rilievi dunali sono stati smembrati in questi ultimi anni per far posto ai nuovi insediamenti turistici di Lignano e Bibione. Inoltre, il cordone litorale più esterno rilevato nel 1973 (BRAMBATI et al., 1982) con quote di 4-5 m sul l.m.m., risulta oggi completamente scomparso a causa della forte erosione che interessa l'apice del delta (vedi BRAMBATI, 1989). Anche il F. Tagliamento, come ad oriente l'Isonzo, è migrato verso Est. Studi recenti (MAROCCO, 1988) hanno messo in evidenza che il delta si è andato via via formando dopo che una diversione verso Est dell' asta terminale l'aveva fatto gravitare nell'area della laguna di Marano. In precedenza sfociava in un imprecisato punto dell' Alto Adriatico, nell'area della laguna di Caorle.

Tra le foci del Tagliamento e dell'Isonzo si estende il complesso lagunare che impropriamente viene denominato laguna di Marano e Grado, ma che, come sarà meglio specificato in seguito, è costituito da due ambienti lagunari distinti, morfologicamente differenti e formatisi in periodi diversi e con modalità distinte. Il complesso lagunare si sviluppa su un'area di circa 16.000 ettari, per una lunghezza di $32 \mathrm{~km}$ circa e per una larghezza di $5 \mathrm{~km}$. È delimitato dal Golfo di Trieste, dal litorale di Grado, dal cordone di neoformazione dei banchi di sabbia dei Tratauri, d'Orio e d'Anfora, e dal vecchio cordone litorale della laguna di Marano, comprendente le isole di S. Andrea e di Martignano e l'ala sinistra del delta tilaventino (Fig. 7). Comunica con il Golfo attraverso una serie di varchi o di bocche lagunari, oggi quasi totalmente fissate da opere idrauliche. All'interno del si- 


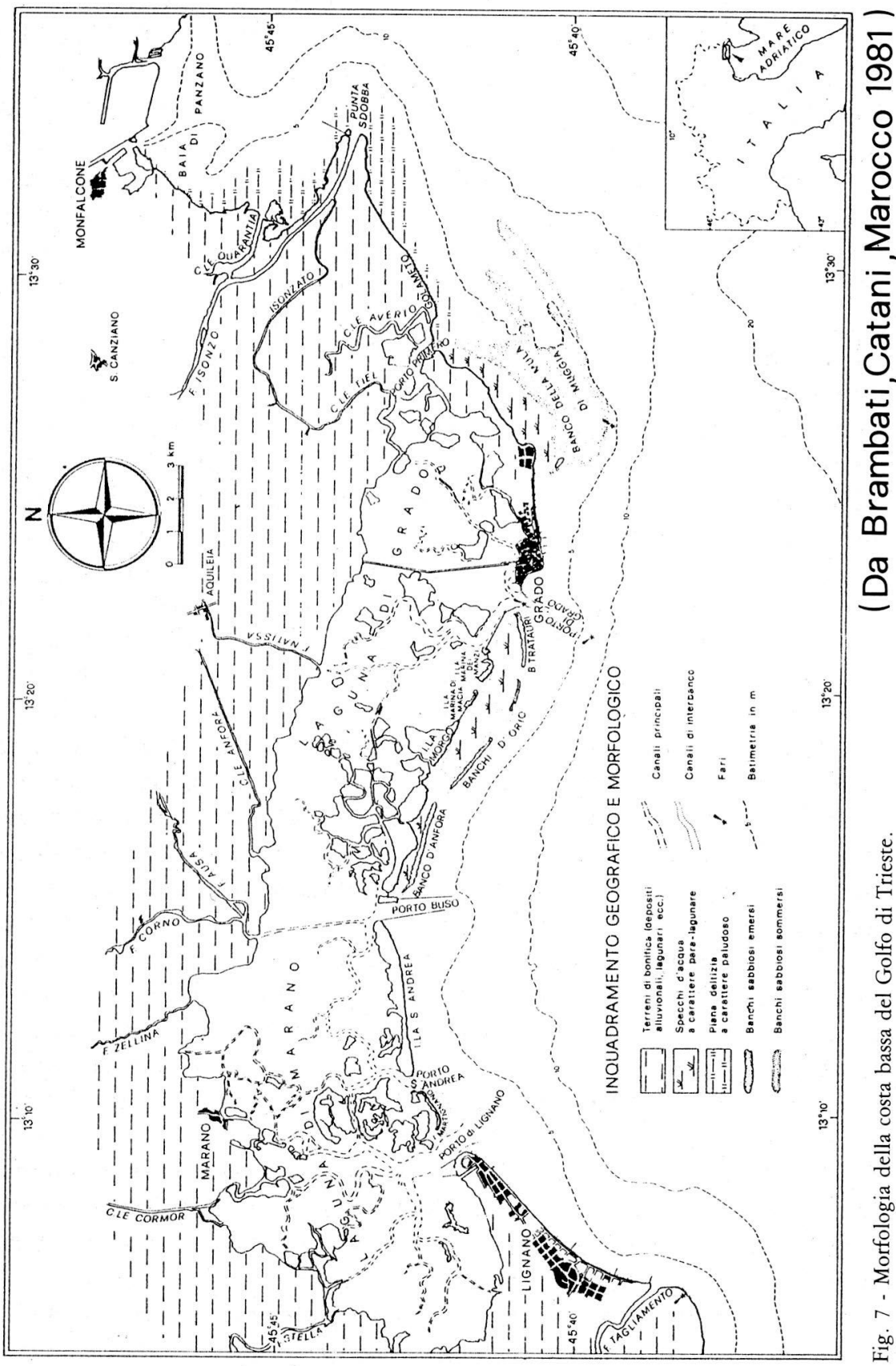




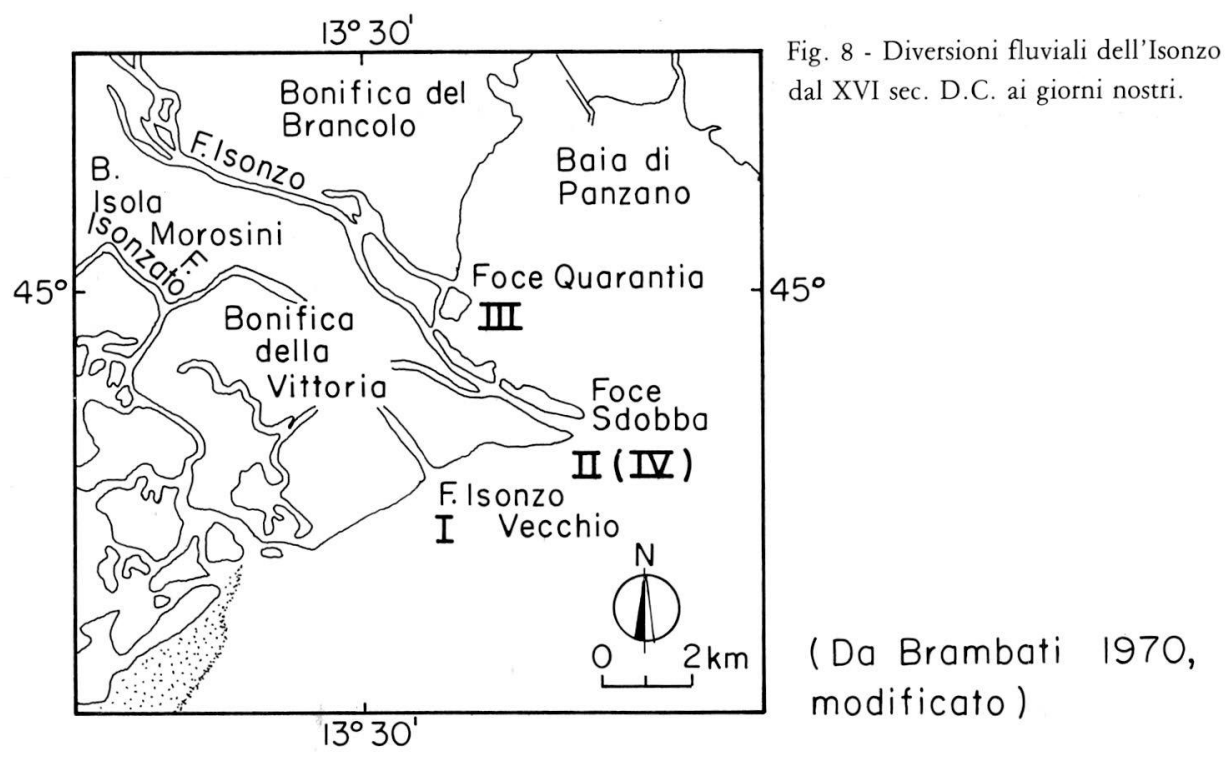

stema lagunare si immettono una serie di fiumi di risorgiva che, ad eccezione del F. Stella, hanno apporto solido ghiaioso-sabbioso molto limitato.

L'attuale ambiente lagunare rappresenta all'incirca il 70\% di quella che era la sua estensione agli inizi del 1800 . E ciò soprattutto per l'opera dell'uomo. Oggi le lagune sono state fissate con arginature al margine interno e dighe o protezioni longitudinali sui cordoni litorali; le bocche sono state in parte sbarrate e, quelle rimaste, regimate con opere idrauliche e continue escavazioni. Canali navigabili vengono continuamente dragati e risagomati in funzione delle nuove esigenze del polo industriale dell'immediato retroterra lagunare, creato ad immagine dell'insediamento di Marghera nella laguna di Venezia. D'altra parte, già all'inizio della prima guerra mondiale, era stato approfondito ed ultimato il canale artificiale della Litoranea Veneta (che unisce per vie d'acqua Venezia con l'Isonzo e Trieste) che taglia longitudinalmente le lagune.

In sostanza, semplificando notevolmente il problema, si può dire che l'ambiente lagunare, ritenuto uno dei pochi ambienti completamente naturali esistenti nella bassa pianura friulana, risulta oggi solamente libero di evolversi in uno spazio predisposto.

Riprendendo e aggiornando la suddivisione areale delle lagune suggerita da BRAMBATI (1969), ma soprattutto da ALBANI, FAVERO \& 


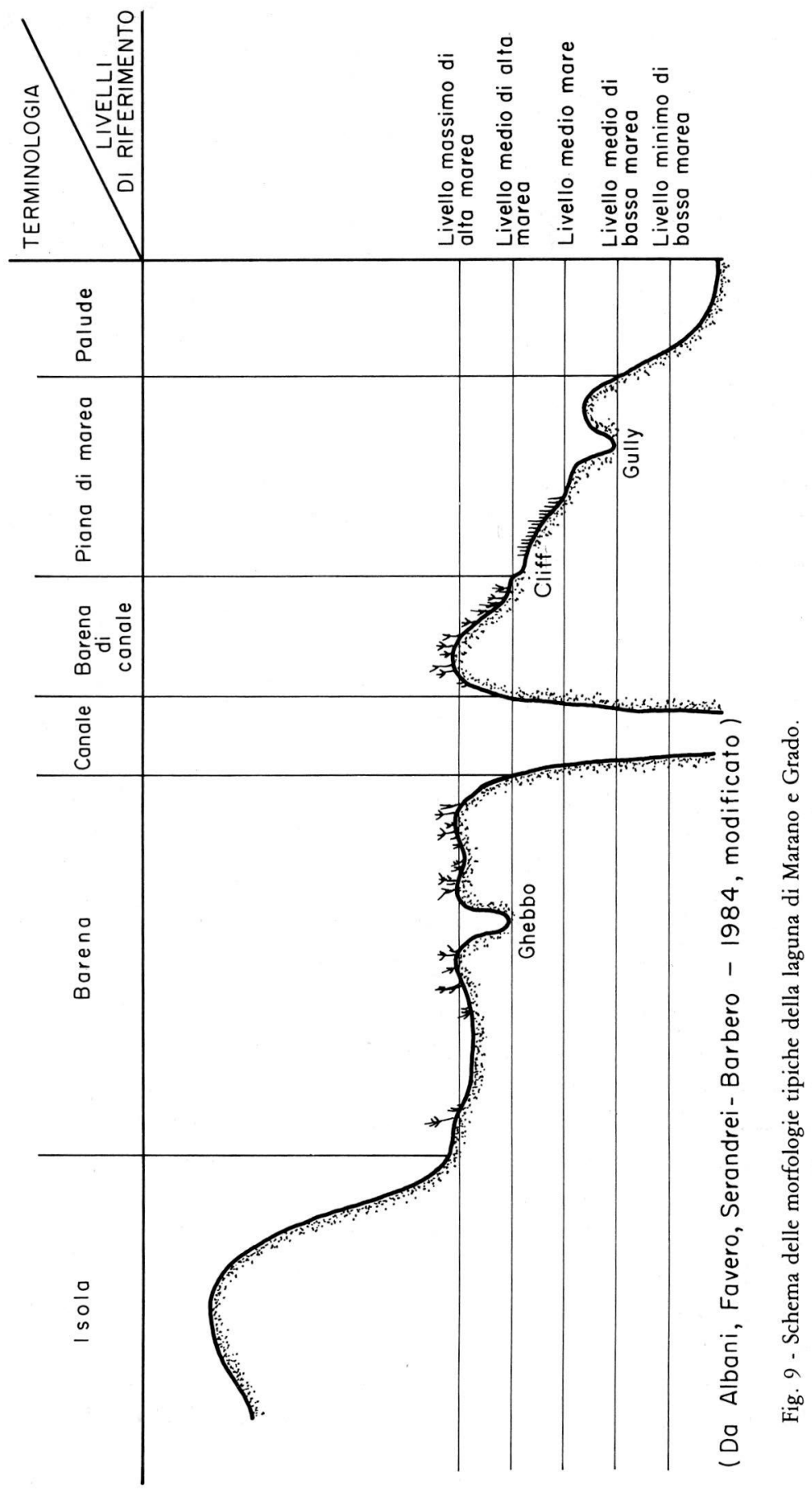


SERANDREI-BARBERO (1984) si possono riconoscere le seguenti morfologie distinte in funzione del livello marino (Fig. 9):

A) morfologie ubicate al di sopra del livello medio delle alte maree;

B) morfologie comprese tra i livelli medi delle alte e basse maree;

C) morfologie poste al di sotto del livello medio delle basse maree;

Prima zona A): comprende forme naturali e artificiali/antropiche.

Tra le prime ricordiamo:

- isole, alti morfologici costituiti da sabbie continentali a volte cementate, presenti nella sola laguna di Grado. Rappresentano la diretta continuazione verso mare dei rilievi dunali che si trovano alle spalle della laguna (Dune di Belvedere, S. Marco, Centenera, ecc.).

- barene, aree colonizzate da una fitta vegetazione di Spartina maritima (ai margini), Limonium vulgare e Salicornia veneta. Anch'esse presenti soprattutto nella laguna di Grado e ubicate di regola ai lati dei canali lagunari (vedi Fig. 9). Si distinguono ulteriormente in barene di piana di alta marea, con classica depressione al centro e a volte interessate da piccoli canali meandriformi (ghebbi) con livello di base corrispondente al livello medio delle alte maree, e da barene di canale, veri e propri argini naturali dei canali lagunari. Sono inoltre presenti alle spalle dei cordoni litorali intorno alle isole e ai piedi delle dighe. Contrariamente a quanto supposto, raramente si trovano in coincidenza di spartiacque dei bacini lagunari;

- cordoni litorali, con profilo tipico (BRAMBATI, 1974) che vede, dal mare verso laguna, la successione del terrazzo di bassa marea, spiaggia, duna e alle spalle le barene (Fig. 10).

Tra le seconde ricordiamo:

- "mote", isole artificiali, rialzate 2-3 m dal l.m.m. con sopra il tipico "casone" di canne e il tamericio;

- argini, che contornano tutto il margine lagunare interno e le numerose valli da pesca;

- terrapieni, il più importante dei quali unisce il retroterra lagunare con l'abitato di Grado;

- dighe, moli, ecc., a difesa delle principali vie di navigazione;

- cumuli di materiali di natura diversa (pseudo-barene), che vengono a volte confusi con le barene (da cui differenziano oltre che per la quota, anche per la stratigrafia) ubicati a fianco dei canali artificiali e di recente bonifica. Rappresentano l'accumulo dei materiali escavati dai canali 


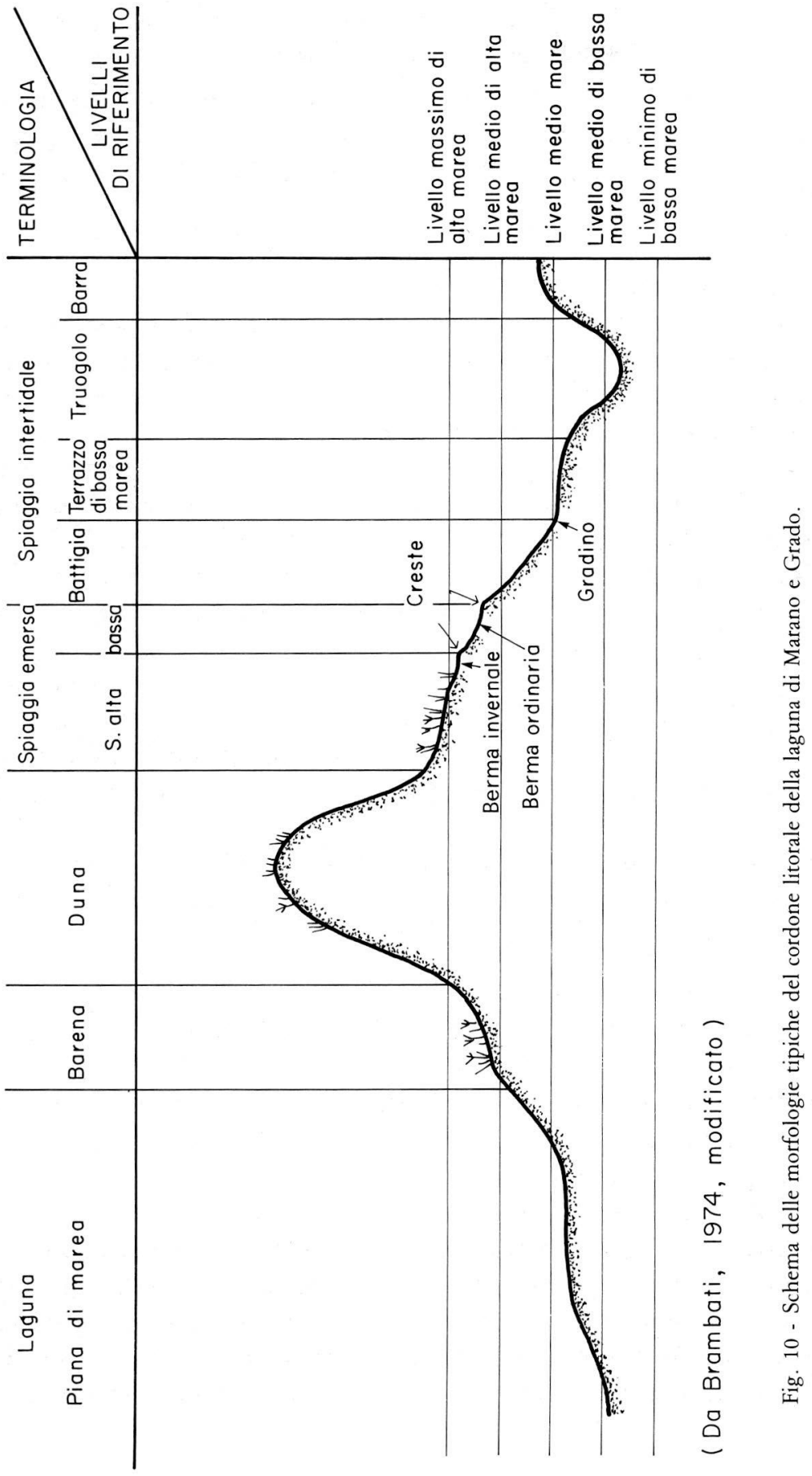


che vengono demoliti e progressivamente rimodellati dalle onde dei natanti.

Seconda zona B): comprende le piane di marea e i canali secondari (dead end, gully), che insieme rappresentano le morfologie a maggior sviluppo areale nella laguna.

- piane di marea (velme), aree pianeggianti a debolissima pendenza che si raccordano con le barene a volte con un gradino di erosione caratteristico con alla base ciottoli di fango. Sono colonizzate da fanerogame marine (Ruppia maritima) e angiosperme Zoostera marina (in prossimità di acque dolci) e Zoosterella noltii. Le piane prive di vegetazione presentano una associazione bentonica tipica rappresentata da forme euriterme ed eurialine (Cerastoderma glaucum, Abra ovata, Venerupis aurea, ecc.);

- canali secondari ( $g u l l y)$, hanno spesso forma meandriforme e si immettono nei canali di ordine superiore con angoli di circa $90^{\circ}$. La linea di base coincide con il livello medio delle basse maree.

Terza zona C): comprende i canali principali (naturali e artificiali) e le paludi (fondao).

- canali si possono distinguere in tre ordini: i canali che congiungono una bocca lagunare con la foce di un fiume immissario in laguna, i canali che si esauriscono nei bacini lagunari o paludi e le loro diramazioni principali. I primi hanno quasi sempre forma rettilinea (soprattutto se artificiali) e sono per lo più normali alla direzione del cordone litorale. I secondi e i terzi sono meandriformi nel loro tratto iniziale e tendono alla linearità in quello terminale. La loro direzione è quasi sempre NW-SE o NE-SW.

- paludi (fondao) rappresentano le aree di depressione con profondità di 1-2 m sotto il livello marino, quindi le zone di laguna che, canali esclusi, rimangono sommerse anche durante le minime maree. Sono ubicati generalmente nella parte settentrionale delle lagune, a ridosso del margine lagunare interno, e sono maggiormente rappresentate nella laguna di Marano.

La sedimentazione all'interno della laguna (Fig. 4) avviene soprattutto ad opera delle correnti di marea. Così le bocche lagunari sono caratterizzate da sedimenti sabbiosi in quanto in questi siti le correnti di marea raggiungono le massime velocità $(1-2 \mathrm{~m} / \mathrm{sec})$; procedendo lungo i canali verso l'interno i sedimenti sabbiosi diminuiscono di percentuale e vengono progressivamente sostituiti da sedimenti pelitici, riflettendo la diminuzione 
della velocità di corrente che si registra progressivamente all'interno della laguna. Questa diminuzione delle dimensioni dei sedimenti che caratterizzano il fondo lagunare, non si registra solamente in funzione dell'aumento della distanza dalla bocca lagunare, ma anche trasversalmente ai canali lagunari via via che si proceda dall'argine alla piana di marea e alla palude. Va sottolineato che ancor oggi l'esatta modalità di trasporto e sedimentazione del particellato che si muove in sospensione nell'ambiente lagunare non è perfettamente conosciuta. Il meccanismo del settling and scour lag effects non sembra dar completamente ragione all'accumulo dei materiali fini all'interno della laguna (BRAMBATI et al., 1983).

A conclusione di questo capitolo preme sottolineare che in questi ultimi anni si stanno svolgendo ricerche che tendono a quantificare e a qualificare le principali morfologie lagunari in funzione dell'idrodinamismo ambientale. Altre ricerche tendono a verificare l'evoluzione dell'ambiente almeno negli ultimi 200 anni. In questo ambito particolare impegno è stato rivolto alla definizione dell'estensione areale delle zone barenicole, delle geometrie idrauliche dei canali lagunari e dei loro rapporti con la superficie ed il volume di svaso-invaso lagunare. E ciò soprattutto per tentare di valutare quali conseguenze sul regime idraulico-sedimentologico delle lagune ha comportato l'intervento antropico e quali si debbano ritenere naturali e quindi insite al sistema.

\section{LA TRASGRESSIONE POSTWÜRMIANA E LE VARIAZIONI DELLA LINEA DI RIVA}

L'argomento è stato più volte trattato da numerosi autori che riprendono in sostanza le ricostruzioni paleogeografiche del Golfo di Trieste eseguite soprattutto sulla base delle conoscenze morfologiche dei fondali (MOSETTI \& D'AMBROSI, 1966) e di quelle litologico-sedimentologiche degli stessi (BRAMBATI \& VENZO, 1969; BRAMBATI, 1970). Ultimamente datazioni assolute $\left({ }^{14} \mathrm{C}\right)$ effettuate nell'area della laguna di Marano e Grado (MAROCCO 1988, 1989) e sul Carso Triestino, abbinate ad analoghe indagini condotte sulla costa istriana del Golfo di Trieste (OGORELEC et al., 1981, 1984; FAGANELI et al., 1987), permettono di delineare i principali momenti evolutivi del Golfo e soprattutto di inquadrarli nell'ambito delle vicissitudini dell'Alto Adriatico.

Attingendo ai risultati di questa ricerca di prossima pubblicazione (MAROCCO, in stampa) si evince che durante l'ultima fase della glaciazione wurmiana (18.000-23.000 anni B.P.), il Golfo di Trieste era un'am- 
pia piana alluvionale, disseminata di acquitrini, dove si accumulavano sedimenti pelitici torbosi o torbe, con una vegetazione di tipo steppico. Le alluvioni dipendevano soprattutto dai fiumi del sistema Isonzo-NatisoneTorre. Durante il Postglaciale cessa la fase di alluvionamento dell'alta paleopianura, $\mathrm{i}$ fiumi incidono i loro vecchi depositi per deporre i materiali così ottenuti nella bassa paleopianura, mentre nella parte più meridionale della stessa inizia la progressiva ingressione marina.

Con la trasgressione Postglaciale il mare raggiunge per primo la costa istriana (circa 10.000 anni B.P.), quindi quella del Carso Triestino (circa 7.000 anni B.P.) e solo ultimamente la costa bassa veneto-friulana. Qui il F. Tagliamento è gravitato, per diversione verso Est, aprendosi un varco sulla preesistente laguna di Marano (presente nell'area da almeno 5.540 anni, quando il livello del mare era più basso dell'attuale di circa $6 \mathrm{~m}$ ) e si è accresciuto formando il suo apparato deltizio in circa 2.000 anni. Più ad Est, la laguna di Marano è andata via via evolvendosi, con un traslazione sulla piana alluvionale contigua, raggiungendo circa 1.400 anni fa, una configurazione molto simile all'attuale. Ancora più ad Est, la laguna di Grado era terraferma solcata da fiumi del sistema isontino, quando gli ambienti deltizi e lagunari occidentali erano già formati. Infatti essa è più giovane della laguna di Marano di circa 4.000 anni e del delta del F. Tagliamento di circa 900 anni. La sua parte occidentale si è formata in epoca tardo-romana in seguito alla diversione verso Est del paleoIsonzo; quella orientale è ancora più recente in quanto legata alle ultime divagazioni verso Est del F. Isonzo, avvenute a cavallo tra il IX e il XVIII secolo D.C.

A conclusione di questo capitolo e al di là delle ulteriori verifiche che dovranno essere intraprese per meglio definire le vicissitudini costiere del Golfo di Trieste nell'Olocene, si sente il dovere di sottolineare che grande importanzą nell'evoluzione del territorio hanno avuto le diversioni verso Est dei fiumi Tagliamento e Isonzo, avvenute in epoche successive e che, almeno per il fiume Isonzo, sono continuate fino ai giorni nostri. Come già evidenziato in lavori precedenti (MAROCCO, 1988 e 1989) non si conoscono i motivi di queste diversioni fluviali; l'ipotesi che sta prendendo sempre più piede è. la diretta conseguenza della progressiva variazioni di gradiente della bassa pianura e del mare prospiciente a causa di movimenti neotettonici lungo le principali linee di dislocazione riconosciute nel sottosuolo.

Analoghi problemi si pongono per la coste alta del Carso Triestino e dell'Istria, in quanto l'esatta influenza della neotettonica nella formazione ma soprattutto nell'evoluzione della costa non ci è ancora nota. Anche questi sono problemi che dovranno essere risolti in un prossimo futuro. 


\section{BIBLIOGRAFIA}

ALBANI A., FAVERO V. AND SERANDREI BARBERO R. 1984: Apparati intertidali ai margini di canali lagunari. Studio morfologico, micropaleontologico e sedimentologico. Ist. Ven. Scienze, Lett. Art, X, 137-162.

Albrecht P. AND MosetTI F. 1987: Karst evolution and sea level. Mem. Soc. Geol. It., 4, 383-387.

Baldi A., Papucci C., Pentassuglia G., Ventura G.C., Giacomelli R., De Guarini F. AND MARASSI G. (in stampa): Distribution of artificial radionucleides in North Adriatic coastal sediments.

Boldrin A. 1979: Aspetti ecologici delle formazioni rocciose dell'Alto Adriatico. Convegno.P.F. Oceanogr. Fondi Ocean., Roma, 1197-1207.

BRAMBATI A. 1969: Sedimentazione recente nelle lagune di Marano e di Grado (Adriatico Settentrionale). St. Trent. Sc. Nat., 46, 142-239.

Brambati A. 1970: Provenienza, trasporto e accumulo dei sedimenti recenti nella laguna di Marano e di Grado e nei litorali tra i fiumi Isonzo e Tagliamento. Mem. Soc. Geol. Ital., 9, 281-329.

BrAmbati A. 1974: Regime sedimentologico delle spiagge dei Banchi d'Oro (Laguna di Grado). Osservazioni dal settembre 1970 all'ottobre 1972. Pubbl. n. 24 del Mus. Friul. St. Nat., Udine, 3-90.

Brambati A. 1989: L'erosione dei litorali. Conv. Geografia nelle Scuole, 34, 1-13.

BRAMBATI A. AND CATANI G. 1988: Le coste e i fondali del Golfo di Trieste dall'Isonzo a Punta Sottile: aspetti geologici, geomorfologici, sedimentologici e geotecnici. Hydrores Information, 6, 13-28.

Brambati A., Catani G., Lenardon G. AND Marocco R. 1982: Rilievi sul litorale da Monfalcone alla foce dell'Adige. Tip. Naz. Trieste.

BRAmbati A., CATANi G. AND MAROCCo R. 1981: Il litorale sabbioso del Friuli-Venezia Giulia: trasporto, dispersione e deposizione dei sedimenti della spiaggia sottomarina. Boll. Soc. Adr. Sc., LXV, 1-32.

Brambati A., Ciabatti M., FAnZutti G.P., Marabini F. AND Marocco R. 1988: Carta sedimentologica dell'Adriatico Settentrionale. P.F. Oceanografia e Fondi Marini. Ist. Geogr. De Agostini Novara.

BRAMBATI A., FANZUTTI G.P. AND MAROCCO R. 1983: Suspended matter transport in lagoons: The Grado Lagoon. Bol. Ocean. Teor. Appl. 1, 5-18.

BRAmBATI A. AND Venzo G.A. 1967: Recent sedimentation in the Northern Adriatic Sea between Venice and Trieste. St. Trent. Sc. Nat., 44, 202-274.

CAVALERI L. AND STEFANON A. 1980: Bottom features due to extreme metereological events in the northern Adriatic Sea. Mar. Geol., 36, 49-64.

DESIO A. 1922: Le variazioni della foce del fiume Isonzo. Riv. Geog: It., 29, 249-268.

FAgANELI J., OGORELEC B., Misic M., DOlENEC T. AND PEZDIC J. 1987: Organic geochemistry of two $40 \mathrm{~m}$ sediment cores from the Gulf of Trieste (Northern Adriatic). Estuarine, Coastal and Shelf Science, 25, 157-167.

FAVERO V. AND PASSEGA R. 1975: Quaternary sedimentation controlled by subsidence, environment, glaciations well CNR Venice-1 Italy. IXeme Congres Intern. de Sedim., Nice, Theme 1, 73-78.

FERUGLIO E. 1936: Sedimenti marini nel sottosuolo della bassa pianura friulana. Boll. Soc. Geol. Ital., LV, 129-138. 
FINETTI I. 1967: Ricerche sismiche a rifrazione sui rapporti strutturali fra il Carso ed il Golfo di Trieste: Boll. Geof. Teor. Appl., 35, 214-225.

LIPPARINI T. 1936: Fossili di sedimenti marini nel sottosuolo della bassa pianura friulana. Boll. Soc. Geol. Ital., 1, 139-151.

MAROCCO R. 1988: Considerazioni sedimentologiche sui sondaggi S19 e S20 (Delta del Fiume Tagliamento). Gortania, Atti Mus. Friul. Sc. Nat., 10, 101-120.

MAROCCO R. 1989: Evoluzione quaternaria della laguna di Marano (Friuli-Venezia Giulia). Il Quaternario, 2, 125-137.

MAROCCO R. (in stampa) Radiocarbon age and Pleistocene-Holocene evolution of Gulf of Trieste coast.

MAROCCO R., TUNIS G. AND VIO E. (in stampa) The transport of sediments along the transect of the Gulf of Trieste during the August 1988 algal bloom. Boll. Oceanol. Teor. Appl.

MARTINIS B. 1953: Le formazioni quaternarie del sottosuolo di Grado (GO). Riv. Ital. Paleont. Stratigr., LIX, 3-22.

MARTINIS B. 1956: Osservazioni stratigrafiche sul sottosuolo di Lignano (Udine). Riv. It. Pal. Strat., 63, 159-178.

MOSETTI F. 1966: Morfologia dell'Adriatico Settentrionale. Boll. Geofis. Teor. Appl., 8, 138-150.

MOSETTI F. 1980: Su due pozzi profondi nella Piana isontina: risultati preliminari delle ricerche Acega per il nuovo acquedotto di Trieste. Boll. Soc. Adr. Sc., LXIV, 7-16.

MoseTtI F. AND D'AMBrosi C. 1966: Cenni sulle vicissitudini costiere dell'Alto Adriatico dedotte dalla attuale morfologia del fondo marino. Atti e Memorie della Commissione Grotte "E. Boegan”, VI, 19-31.

NEWTON R.S. AND STEFANON A. 1982: Side-scan sonar and subbottom profiling in the northern Adriatic Sea. Mar. Geol., 46, 279-306.

Ogorelec B., Misic M., Sercely A., Cimerman F., Faganeli J. And Stegnar P. 1981: The sediment of the salt marsh of Secovije. Geologija, 24, 179-216.

Ogorelec B., Misic M., Faganeli J., Sercelj A., Cimerman F., Dolenec T. and Pezdic J. 1984: Quaternary sediment from the borebole $V-3$ in the Bay of Koper. Slovensko morje in zaledje 6/7, 165-186.

ROSSI B., MOSETTI F. AND CESCON B. 1968: Morfologia e natura del Golfo di Trieste (Adriatico Settentrionale fra Punta Tagliamento e Punta Salvore). Boll. Soc. Adr. Sc., 2, 187-206.

SPENCER T. 1988: Limestone coastal morphology: the biological contribution. Progress in Physical Geography, 12, 66-111.

STEFANON A. 1984: Sedimentologia del Mare Adriatico: rapporti tra erosione e sedimentazione olocenica. Boll. Ocean. Teor. Appl., 4, 281-324.

STEFANON A. 1985: Marine sedimentology through modern acoustical methods: 1 . side scan sonar. Boll. Ocean. Teor. Appl., 1, 3-38.

TORUNSKI H. 1979: Biological erosion and its significance for the morphogenenis of limestone coasts and for nearshore sedimentation. Senckenbergiana maritima, 11, 193265.

TUNIS G., CATANI G. AND STEFANI E. 1982: Una nuova trappola batimetrica per lo studio del trasporto dei sedimenti in sospensione in prossimità della linea di riva. Boll. Soc. Adr. Sc., 66, 17-31. 